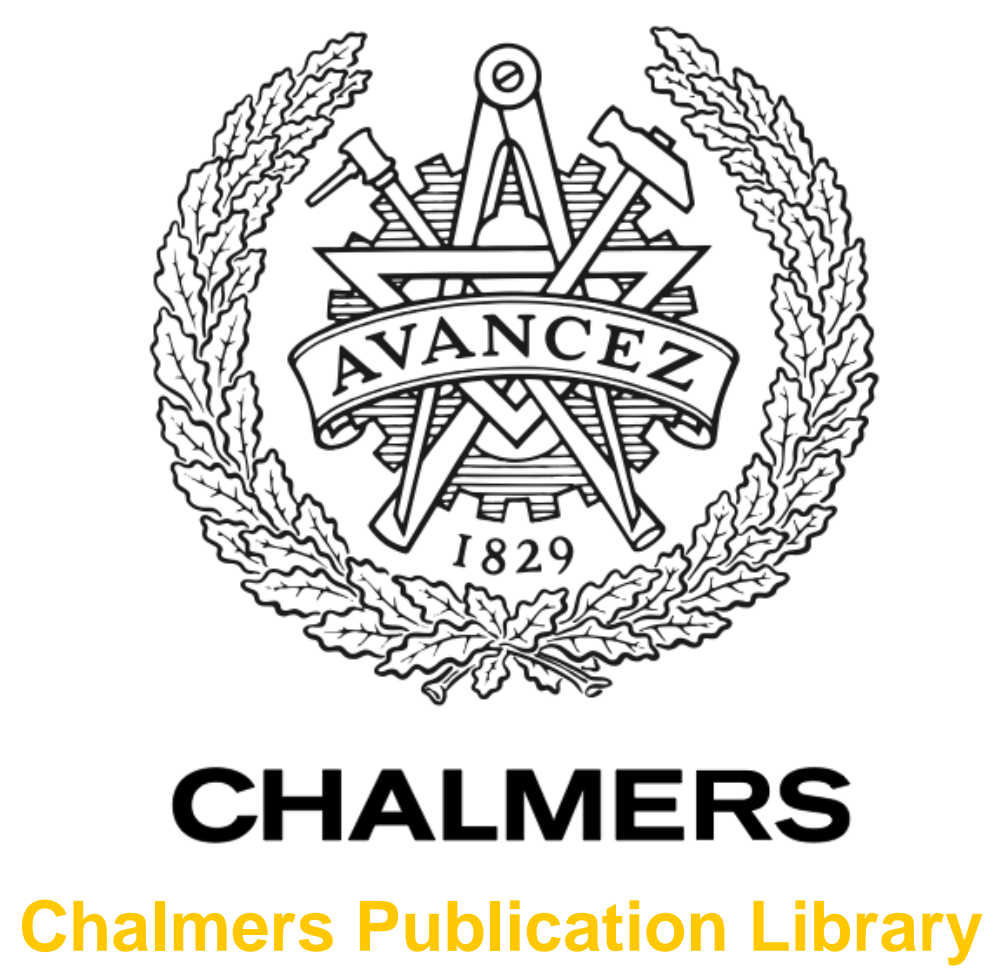

Sensing or Transmission: Causal Cognitive Radio Strategies with Censorship

This document has been downloaded from Chalmers Publication Library (CPL). It is the author's version of a work that was accepted for publication in:

IEEE Transactions on Wireless Communications (ISSN: 1536-1276)

Citation for the published paper:

Haghighi, K. ; Ström, E. ; Agrell, E. (2014) "Sensing or Transmission: Causal Cognitive Radio Strategies with Censorship". IEEE Transactions on Wireless Communications, vol. 13(6), pp. 3031-3041.

Downloaded from: http://publications.lib.chalmers.se/publication/211155

Notice: Changes introduced as a result of publishing processes such as copy-editing and formatting may not be reflected in this document. For a definitive version of this work, please refer to the published source. Please note that access to the published version might require a subscription. 


\title{
Sensing or Transmission: Causal Cognitive Radio Strategies with Censorship
}

\author{
Kasra Haghighi, Member, IEEE, Erik G. Ström, Senior Member, IEEE, and Erik Agrell, Senior Member, IEEE
}

\begin{abstract}
This paper introduces a novel opportunistic transmission strategy for cognitive radios (CRs). The primary user (PU) is assumed to transmit in a time-slotted manner according to a two-state Markov model, and the CR is either sensing, that is, obtaining a causal, noisy observation of a primary user (PU) state, or transmitting, but not both at the same time. In other words, the CR observations of the PU are censored whenever the $C R$ is transmitting. The objective of the $C R$ transmission strategy is to maximize the utilization ratio (UR), i.e., the relative number of the $\mathrm{PU}$-idle slots that are used by the CR, subject to that the interference ratio (IR), i.e., the relative number of the PU-active slots that are used by the CR, is below a certain level. We introduce an a-posteriori LLR-based CR transmission strategy, called CLAPP, and evaluate this strategy in terms of the achievable UR for different PU model parameters and received signal-to-noise ratios (SNRs). The performance of CLAPP is compared with a simple censored energy detection scheme. Simulation results show that CLAPP has $52 \%$ gain in UR over the best censored energy detection scheme for a maximum IR level of $10 \%$ and an SNR of $-2 \mathrm{~dB}$.
\end{abstract}

Index Terms-Spectrum utilization, interference ratio, spectrum sensing, cognitive radio, hidden Markov model, opportunistic spectrum access, DSA, missing observation, censorship, CLAPP.

\section{INTRODUCTION}

$\mathbf{P}$ ROLIFERATION of smartphones and hand-held devices has elevated the demand for high speed wireless services. In the United States alone, as part of the national wireless initiative, there are plans to bring wireless broadband internet access to $98 \%$ of the Americans [1]. This desire for high datarate wireless networking creates huge expectations for more frequency spectrum. However, spectrum is a scarce commodity, which is mostly licensed to certain operators commonly known as primary users (PUs) of the band, who spent a considerable amount of investment in retaining the right to use these bands uninterrupted. Notably, this valuable resource is severely under-utilized [2] and many spectrum holes exist in both the time and spatial domains. Due to their great scope of use and benefits, there is significant interest in techniques providing opportunistic secondary spectrum access [3], [4];

This work was supported in part by the High Speed Wireless Communication Center, hosted by Lund University, and the Swedish Foundation for Strategic Research. The calculations were performed in part on resources provided by the Swedish National Infrastructure for Computing (SNIC) at C3SE.

K. Haghighi was with the Department of Signals and Systems, Chalmers University of Technology, SE-41296 Göteborg, Sweden, and is now with UniqueSec AB (email: kasra@ieee.org).

E. G. Ström and E. Agrell are with the Department of Signals and Systems, Chalmers University of Technology, SE-41296 Göteborg, Sweden (email: erik.strom@chalmers.se, agrell@chalmers.se). these are collectively termed cognitive radio (CR). CRs adapt to exploit communication opportunities in the spectrum by making use of it without interfering with legitimate users.

An enabler for dynamic spectrum reuse by the $\mathrm{CR}$ is agile and reliable spectrum sensing [5], which means to estimate when the PU is not transmitting in the licensed band. There have been several attempts to design spectrum sensing schemes that do not need to have a model for PU transmissions. A straightforward approach is energy detection, which simply means to sum up the energy of received samples and compare with a threshold [6]. However, energy detection performance is limited by the signal-to-noise (SNR) wall, which is the SNR below which robust detection is impossible for the given detector [7], due to the low received power of the PU signal at the CR receiver, as well as uncertainties in signals, noise, and channel, which ultimately result in large sensing delays. In wideband spectrum sensing in particular, the tradeoff between agility and reliability is more noticeable [8], [9]. This creates a demand for a CR which uses all previous observations and makes a transmission decision with the shortest possible delay. Sequential spectrum sensing methods, which collect samples sequentially until one of two thresholds is met, are attractive, since they are on average faster than standard energy detection [6], [10], [11].

In contrast to the models which ignore dependencies between PU transmissions, measurement campaigns and recent studies [12] have shown that hidden Markov models (HMM) fit the PUs behavior in many different bands. Assuming a Markov model for PU activities provides better reutilization of the spectrum whilst being representative of reality and is used in many CR research papers [13]-[19]. To be able to use HMMs for modeling the PU behavior, the knowledge of model parameters is necessary. The impact of model parameters estimation on the CR performance was investigated in [20], [21], which, overall, appears to be quite promising. Another PU models with finite backlog is considered in [22].

To exploit the Markov model, the Markov decision process [13]-[16] and the partially observed Markov decision process [17], [19] are widely used. Moreover, there exist works in the prediction of the future state of the PU [18], [23]-[25]. In our previous work, we have introduced an optimum causal strategy that utilizes this PU behavior [26], [27] with low complexity. This approach not only considers the PU transmission model but also takes the causality of observations into account and thus provides a better reutilization of the spectrum.

In some CR systems, transmission and reception in the same frequency band at the same time are not possible because at a $\mathrm{CR}$ receiver, the signal transmitted by the same CR will 
be much stronger than the received PU signal, which the spectrum sensing mechanism is supposed to detect. To avoid this, the optimization of the sensing time vs. transmission time was considered in [28]-[33]. In [34], the energy consumption of sensing was also considered and attempts were made to minimize it. These methods have considered a fixed length of sensing and transmission. In another interesting publication [35], the probability of detection of the PU was considered as the constraint, under which the secondary rate was optimized. The same authors presented efficient spectrum sensing for CR networks via joint optimization of sensing threshold and duration in [36].

In this paper, we extend the findings in [27] and use HMMs as a tool for better sensing and transmitting in spectrum holes. We introduce a CR strategy which can either transmit or sense, but not both at the same time. In this strategy, the sensing is performed as long as it is necessary and then a transmission lasts as long as it is safe enough to transmit. The PU signal is considered missing, or "censored," during transmission. Furthermore, we introduce a simple and iterative method to calculate a test statistic from the observations with missing PU samples, where, in contrast to [37], the missing observations are dependent on the previous transmission pattern.

Our main contributions are summarized as follows.

- We propose a realistic model, which considers spectrum sensing with missing observations (due to CR transmission)

- We introduce a method for calculating a-posteriori probabilities log-likelihood ratio (APP-LLR) for the future PU transmission from the observation with censorship, which depends iteratively on previous observations

- A novel transmission strategy for a CR with censored spectrum observations is established.

The main differences with our previous work in [27] results from the censorship. In particular, since the censorship is dependent on previous transmission decisions, the LLR statistics and its cumulative distribution function (CDF) varies over time. Hence,

- a new method to calculate the LLRs is needed, and

- the calculation of the threshold is quite different, since the empirical CDF of the LLRs cannot be used.

Moreover, this paper analyses the performance of the strategies in certain degenerate cases not found in [27].

\section{SYSTEM MODEL}

A cognitive communication link consists of a CR transmitter-receiver pair and the channel in between. In this paper, we are evaluating the interaction between a single PU and single CR. The wireless channel in between is assumed to be known.

This section presents the abstract system model as depicted in Fig. 1, which accounts for the PU signal, CR noise, and $\mathrm{CR}$ reception with censorship. This model demonstrates the PU activity on top, the CR received signal in the middle, and the CR transmission strategy at the bottom. Whenever the CR decides to transmit, the next PU activity is censored. First, a more general perspective is considered and then a simplified version will be used.

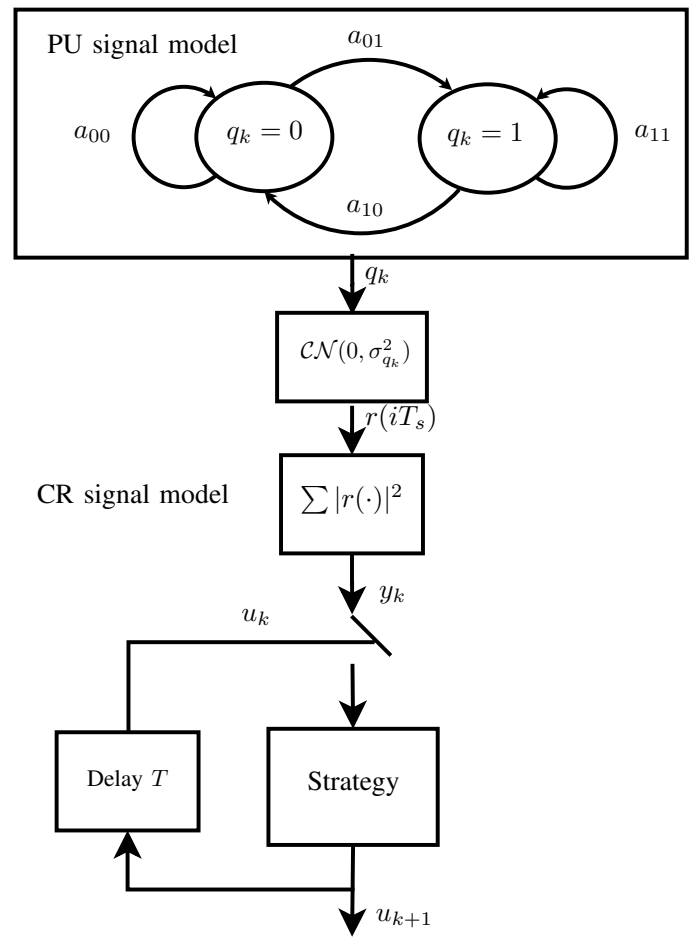

Figure 1. System model; $q_{k}=1$ indicates a PU transmission in the $k$ th slot and $q_{k}=0$ indicates no transmission. The received samples $r\left(i T_{s}\right)$ are circularly symmetric complex Gaussian random variables, whose variance depends on the PU state. The CR uses the energy detector output to decide whether to transmit $\left(u_{k+1}=1\right)$ or not $\left(u_{k+1}=0\right)$ in slot $k+1$. If $u_{k+1}=1$, then the next observation is censored.

\section{A. PU Transmission Model}

A cognitive radio system is designed to utilize spectrum vacancies. To take advantage of time-frequency slots which are not used by the PU, the CR must be aware of the PU activity model. The CR estimates this by collecting samples from PU transmissions over a noisy channel.

The PU transmissions are assumed to be slotted, since in most of today's digital communication systems, transmissions are confined within a packet, frame, or generally some block structure of some minimum frame length $T_{\mathrm{F}}$. However, the $\mathrm{CR}$ will model the PU as having a transmission slot length $T$, where $T \ll T_{\mathrm{F}}$. We can think of $T$ as a tuning parameter, whose effect on the CR performance will be explained below. The PU transmission in the $k$ th time slot, i.e., for $t \in[k T,(k+1) T)$, is described by the PU transmission state $q_{k}$, where $q_{k}=1$ and $q_{k}=0$ indicates transmission and no transmission, respectively. For simplicity, we will assume that the time slots are synchronized to the PU transmissions. This is not a very restrictive assumption, since because $T \ll T_{\mathrm{F}}$, a synchronization mismatch will only affect a small fraction of the slots (namely those slots in which the PU starts or ends a transmission). The PU transmission state sequence $q_{k}$, for $k=0,1, \ldots$, is assumed to follow a two-state Markov model with state transition probabilities

$$
a_{i j} \triangleq \operatorname{Pr}\left\{q_{k+1}=j \mid q_{k}=i\right\}, \quad i, j \in\{0,1\},
$$

as depicted in the top part of Fig. 1. We assume that the PU does not remain in the same state forever, i.e., that $a_{i j}>0$ for 
$i \neq j$. Furthermore, we assume that the Markov chain is in steady state at $k=0$, which implies that the state probabilities do not depend on $k$, i.e., that

$$
\begin{aligned}
& \pi_{0} \triangleq \operatorname{Pr}\left\{q_{k}=0\right\}=\frac{a_{10}}{a_{01}+a_{10}}, \\
& \pi_{1} \triangleq \operatorname{Pr}\left\{q_{k}=1\right\}=\frac{a_{01}}{a_{01}+a_{10}}
\end{aligned}
$$

for $k=0,1, \ldots$, [38]. The transition probabilities are assumed to be known or accurately estimated from data, e.g., by using the expectation-maximization algorithm [21], [38].

Since the PU is assumed to have a minimum transmission slot $T_{\mathrm{F}}$, a two-state Markov model with slot length $T \ll T_{\mathrm{F}}$ can only approximate the true PU behavior. The reason for selecting $T \ll T_{\mathrm{F}}$ is to improve the $\mathrm{CR}$ agility, i.e., its ability to quickly sense changes in the PU state and to mitigate the impact of synchronization errors. However, we cannot choose $T$ to be too small, since the resulting Markov model will lose in accuracy and the SNR will be reduced (as explained below). Finally, we note that as a consequence of choosing $T \ll T_{\mathrm{F}}$, the probability that the PU switches states is small, i.e., we can safely assume that $a_{01} \ll a_{00}$ and $a_{10} \ll a_{11}$.

Another factor in modeling the PU-CR interaction is the channel in between. Wireless channels are normally considered as random fading processes such as Rayleigh, Rician, Nakagami, etc. [39], [40]. Another approach to modeling the fading process is to include the fading in the PU transmission model. Thus, whenever the channel is in a deep fade, it is assumed that there is no PU transmission, no matter what the real state of the PU is. Conversely, in case of no deep fade, the standard PU transmission model is used. Thus, a simple two-state Markov model can approximate a wide range of PU transmissions, PU network activities, and even fading channels.

\section{B. Signal and Noise Model}

We model the PU-CR channel as an additive white Gaussian noise (AWGN) channel. The complex envelope of the CR received signal, low-pass filtered to the PU signal bandwidth $W$, is

$$
r(t)=\left\{\begin{array}{ll}
n(t), & q_{k}=0 \\
s(t)+n(t), & q_{k}=1
\end{array}, \quad t \in[k T,(k+1) T),\right.
$$

where $n(t)$ is the filtered AWGN channel noise and the contribution from the PU transmitted signal, $s(t)$, is modeled as a circularly symmetric complex Gaussian random process with bandwidth $W$. This PU signal model is common in the literature [41] [4], and is reasonable for many combinations of PU signal formats and channels (fading as well as nonfading). If we select the sample interval $T_{s}$ such that $T_{s} \gg 1 / W$, then the samples in the $k$ th slot can be approximated as independent, identically distributed (i.i.d.) complex Gaussian random variables

$$
\begin{aligned}
r\left(k T+i T_{s}\right) & =n\left(k T+i T_{s}\right)+s\left(k T+i T_{s}\right) \\
& \sim\left\{\begin{array}{ll}
\mathcal{C N}\left(0, \sigma_{0}^{2}\right), & q_{k}=0 \\
\mathcal{C N}\left(0, \sigma_{1}^{2}\right), & q_{k}=1
\end{array}, \quad i=0,1, \ldots, K_{\max },\right.
\end{aligned}
$$

where $\mathcal{C} \mathcal{N}\left(\mu, \sigma^{2}\right)$ denotes a circularly symmetric, complex Gaussian distribution with mean $\mu$ and variance $\sigma^{2}, K_{\max }=$ $\left\lfloor T / T_{s}\right\rfloor$ with $\lfloor x\rfloor$ being the largest integer not greater than $x, \sigma_{0}^{2}$ is the noise variance, and $\sigma_{1}^{2}=\sigma_{0}^{2}+\sigma_{s}^{2}$ where $\sigma_{s}^{2}$ is the signal power. We define the signal-to-noise-ratio as $\mathrm{SNR} \triangleq \sigma_{s}^{2} / \sigma_{0}^{2}$. For ease of presentation, we fixed $\sigma_{0}^{2}=2$ in the figures and simulations.

Since we do not have knowledge of the PU signal phase, the CR uses an energy detector front-end to form the statistics

$$
y_{k} \triangleq \sum_{i=0}^{K-1}\left|r\left(k T+i T_{s}\right)\right|^{2}
$$

where $K \leq K_{\max }$. Hence, since $y_{k}$ is the sum of the squared magnitude of $K$ i.i.d. complex Gaussian random variables, or equivalently the sum of $2 K$ squared real-valued Gaussian random variables, $y_{k}$ is proportional to a standard Chi-squared random variable with $2 K$ degrees of freedom. To be precise, $y_{k} /\left(\sigma_{0}^{2} / 2\right) \sim \chi_{2 K}^{2}$ if $q_{k}=0$ and $y_{k} /\left(\sigma_{1}^{2} / 2\right) \sim \chi_{2 K}^{2}$ if $q_{k}=1$, where $\chi_{N}^{2}$ denotes a standard Chi-squared random variable with $N$ degrees of freedom. The tuning parameter $K$ essentially determines the SNR in $y_{k}$. Since $K$ must be no greater than $K_{\max }=\left\lfloor T / T_{s}\right\rfloor$, we see that reducing $T$ will eventually limit the maximum SNR.

\section{CR model}

In this paper, it is assumed that the CR always has information to send, i.e., it has a full buffer, and will seek to reuse the spectrum whenever it is available. However, spectrum sensing cannot always be performed, as the CR is not able to observe the spectrum during its transmission periods. This limitation arises from the fact that, in practice, a transmission from a CR transmitter will saturate its receiver and, thus, it will be extremely difficult to sense at the same time in the same frequency band. The CR strategy decides to transmit or sense in each time slot. At time slot $k$, a transmission decision for the next time slot is represented by $u_{k+1}=1$ and a sensing decision is denoted by $u_{k+1}=0$. In this paper, we assume that the transmission strategy has access to the spectrum's energy $y_{k}$ only when $u_{k}=0$. In other words, the CR will observe the list $\mathbf{y}_{k}^{\prime}$,

$$
\mathbf{y}_{k}^{\prime} \triangleq\left\{\begin{array}{ll}
\mathbf{y}_{k-1}^{\prime}, & \text { if } u_{k}=1, \\
{\left[\begin{array}{ll}
\mathbf{y}_{k-1}^{\prime} & y_{k}
\end{array}\right],} & \text { if } u_{k}=0
\end{array}, k=1,2, \ldots,\right.
$$

where $\mathbf{y}_{0}^{\prime}=[]$, i.e., the empty list. Obviously, the length of $\mathbf{y}_{k}^{\prime}$ is smaller than or equal to $k$.

So, the actual observation, which is used by the strategy to make the next transmission decision, is dependent on the previous transmission decisions.

\section{Definition of a $C R$ transmission strategy}

To be able to judge different cognitive radio transmission strategies with censorship, first we need to establish a proper mathematical definition for such a strategy. Our goal is to design the best CR transmission strategy, with the output $u_{k+1}$, where $u_{k+1}=0$ and $u_{k+1}=1$ represent no transmission and 
transmission, respectively, in slot $k+1$ using the observations until time $k, \mathbf{y}_{k}^{\prime}$ as defined in (5).

Now, we formally define the CR transmission strategy as a series of functions $f_{k}(\cdot)$, which produce the transmissions decisions. In other words, a CR transmission strategy is

$$
\begin{aligned}
\mathfrak{F} & =\left(f_{0}, f_{1}, f_{2}, \ldots\right), \\
u_{k+1} & =f_{k}\left(\mathbf{y}_{k}^{\prime}, a_{01}, a_{10}, \sigma_{0}^{2}, \sigma_{1}^{2}\right) .
\end{aligned}
$$

Later, for ease of notation, we will omit the PU model information $\left(a_{01}, a_{10}, \sigma_{0}^{2}, \sigma_{1}^{2}\right)$ and simply denote the decision functions by $f_{k}\left(\mathbf{y}_{k}^{\prime}\right)$. This formal definition of a strategy does however not offer a practically implementable CR algorithm, due to the prohibitive complexity of storing and processing the full history $\mathbf{y}_{k}^{\prime}$. In Sec. III, we will develop a recursive algorithm, which avoids storing $\mathbf{y}_{k}^{\prime}$.

\section{E. Problem Statement}

In wireless communications, cognitive radios are employed to reuse idle spectrum slots by utilizing the spectrum sensing information, whenever possible. The CR has access to observations from the spectrum to decide whether to transmit or not. However, due to the uncertainties in the channel, the noise, and the PU future states, the CR will create unintentional interference for the PU. Interference will happen whenever the CR transmits at the same time as the PU. This interference is quantified by the interference ratio (IR) $\rho$, defined as [26], [27]

$$
\rho \triangleq \operatorname{Pr}\left\{u_{k+1}=1 \mid q_{k+1}=1\right\}
$$

where we have implicitly assumed that $k$ is large enough for the initial transient to have passed and that the system is in steady state, in the sense that $\operatorname{Pr}\left\{u_{k+1} \mid q_{k+1}\right\}$ does not depend on $k$. A CR is supposed not to interfere with the PU more than a specific limit $\rho_{\max }$.

As explained in Sec. II-C, a CR strategy considers observations when sensing is allowed and decides about the next transmission. Utilization of the spectrum occurs whenever the $\mathrm{CR}$ transmits in a vacant time slot, and this is measured by the spectral utilization ratio (UR), defined as [26], [27]

$$
\eta \triangleq \operatorname{Pr}\left\{u_{k+1}=1 \mid q_{k+1}=0\right\}
$$

where we have again assumed that $k$ is sufficiently large.

There are major differences between the system model in this paper and some previous works in the literature, such as [17], [19], [23], [27]. Firstly, we make transmission decisions $u_{k+1}$ based on all previous (causal) observations $\mathbf{y}_{k}^{\prime}$, unlike the methods in [17], [19], [23] which make transmission decisions based on a single observation. Secondly, previous transmission decisions control which energy samples we can observe, which is not the case in [27].

The main difficulty in designing good CR transmission strategies is the dependence of censorship on previous decisions. This feedback, which is visible in Fig. 1, adds another dependence between observations in addition to the PU Markov dependence.

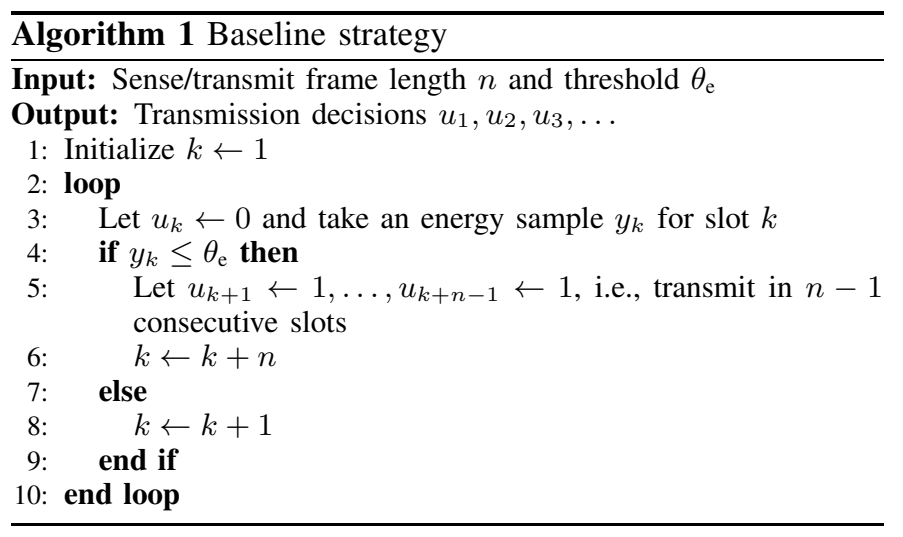

\section{F. Baseline strategy}

The baseline strategy, which is using energy detection and censoring, is explained in Algorithm 1. In this strategy there are two parameters to optimize: $\theta_{e}$ and $n$. These two design parameters should be chosen to maximize $\eta$, subject to the condition $\rho \leq \rho_{\max }$. Moreover, we know that UR and IR are increasing functions of $\theta_{e}$ [26], [27]. Thus, to find optimum parameters for the baseline strategy, we first fix $n$ and find the threshold such that $\rho=\rho_{\max }$ through bisection search. Then we repeat this for different $n$ to maximize the UR. For different $a_{01}, a_{10}, \sigma_{0}^{2}, \sigma_{1}^{2}$, and $\rho_{\max }$, different sets of $\theta_{e}$ and $n$ must be chosen.

However, this strategy has some limitations. By design, the baseline CR transmits for $n-1$ consecutive slots before sensing for at least one slot. Hence, even if the CR transmits as often as it can, i.e., for $n-1$ out of $n$ slots, the UR and IR are upper-bounded: $\eta, \rho \leq(n-1) / n=1-1 / n$. Clearly, we can remove this problem by increasing $n$. However, as $n$ increases, we need to decrease the threshold $\theta_{c}$ to ensure that $\rho \leq \rho_{\max }$, and this will lead to fewer transmissions and a reduced $\eta$. Intuitively, we therefore expect that there exists a finite optimum $n$ for each combination of PU parameters $\left(a_{01}\right.$, $a_{10}, \sigma_{0}^{2}$, and $\sigma_{1}^{2}$ ). This intuition is verified by the numerical results in Sec. IV-B. Hence, the complexity in finding the optimum $n$ for the baseline strategy is not excessive, and is anyways not important, since we are not suggesting to use the baseline strategy in practice.

\section{CEnSORED APP-LLR BASED COGNITIVE TRANSMISSION STRATEGY}

In this section, we introduce a new strategy which observes spectrum energy samples through censorship by its own transmissions. In our previous paper [27], we have shown that the APP-LLR transmission strategy is optimum in an uncensored scenario (i.e., when simultaneous sensing and transmission are allowed). Now, since we are dealing with censored observations, a straightforward direction for designing a CR strategy is to extend the non-censored APP-LLR (NCLAPP) strategy in [27] to the censored APP-LLR (CLAPP $\left.{ }^{\circledR}\right)$ strategy described below. CLAPP (as well as NCLAPP) has the advantages that

- it captures all information about the previous observations recursively,

- it includes the PU Markov model in its decisions, 


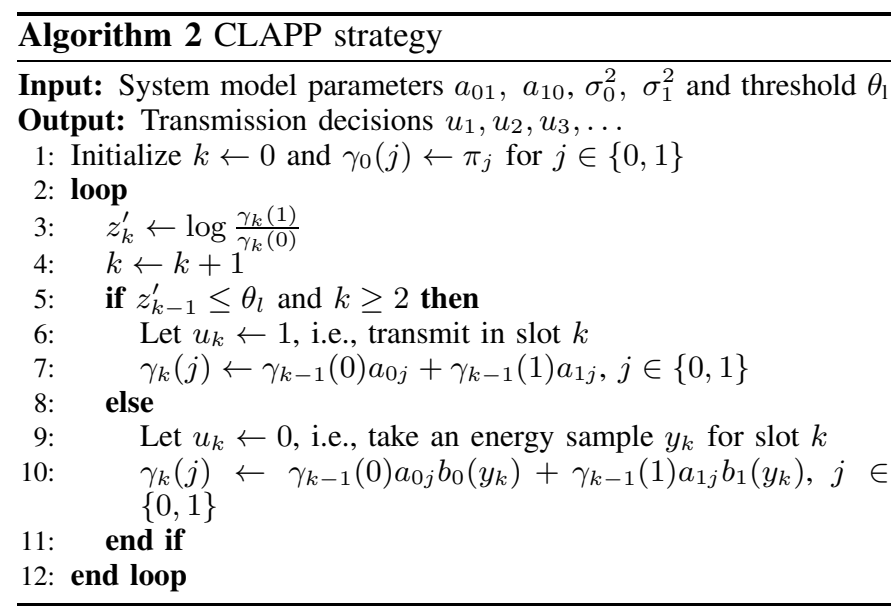

- it predicts the next state of the PU.

At the same time, NCLAPP is very simple to implement.

\section{A. Introduction to the CLAPP strategy}

In our previous contribution [27], the LLRs were calculated based on the forward variables $\alpha_{k}(j)$ of all observations. Specifically, NCLAPP calculates the LLRs as [26, Eqs. (19)(21)]

$$
z_{k} \triangleq \log \frac{\operatorname{Pr}\left\{q_{k+1}=1 \mid \mathbf{y}_{k}\right\}}{\operatorname{Pr}\left\{q_{k+1}=0 \mid \mathbf{y}_{k}\right\}}=\log \frac{a_{01} \alpha_{k}(0)+a_{11} \alpha_{k}(1)}{a_{00} \alpha_{k}(0)+a_{10} \alpha_{k}(1)},
$$

where $\mathbf{y}_{k} \triangleq\left[y_{1}, y_{2}, \ldots, y_{k}\right]$ and $\alpha_{k}(j) \triangleq p\left(q_{k}=j, \mathbf{y}_{k}\right), \quad j \in$ $\{0,1\}$.

The CLAPP algorithm is explained in Algorithm 2. In CLAPP, we have access only to the censored observations $\mathbf{y}_{k}^{\prime}$. Thus, we have to calculate the LLRs based on $\mathbf{y}_{k}^{\prime}$. We first define the censored APP LLRs as

$$
z_{k}^{\prime} \triangleq \log \frac{\operatorname{Pr}\left\{q_{k+1}=1 \mid \mathbf{y}_{k}^{\prime}\right\}}{\operatorname{Pr}\left\{q_{k+1}=0 \mid \mathbf{y}_{k}^{\prime}\right\}}
$$

and then express $z_{k}^{\prime}$ in terms of the joint distributions instead of the conditionals as

$$
z_{k}^{\prime}=\log \frac{p\left(q_{k+1}=1, \mathbf{y}_{k}^{\prime}\right)}{p\left(q_{k+1}=0, \mathbf{y}_{k}^{\prime}\right)}=\log \frac{\gamma_{k}(1)}{\gamma_{k}(0)},
$$

where $\gamma_{k}(j) \triangleq p\left(q_{k+1}=j, \mathbf{y}_{k}^{\prime}\right)$. These censored LLRs capture all the information needed for making decisions based on the censored observations. Clearly, a decision rule of the general form (7) requires enormous amounts of memory for storing all previous censored observations $\mathbf{y}_{k}^{\prime}$. However, Algorithm 2 has the advantage of requiring only the latest observation for making decisions, which is very suitable for real world implementations.

One simple approach in NCLAPP, which was proven to be optimal in terms of $\eta$ in [27], is the comparison of LLRs with a fixed threshold. In CLAPP, we also implement the same approach and compare the censored LLRs with a fixed threshold. The strategy $u_{k+1}=f_{k}\left(\mathbf{y}_{k}^{\prime}\right)$ is thus

$$
f_{k}\left(\mathbf{y}_{k}^{\prime}\right)=\left\{\begin{array}{ll}
1, & \text { if } z_{k}^{\prime} \leq \theta_{l} \\
0, & \text { if } z_{k}^{\prime}>\theta_{l}
\end{array},\right.
$$

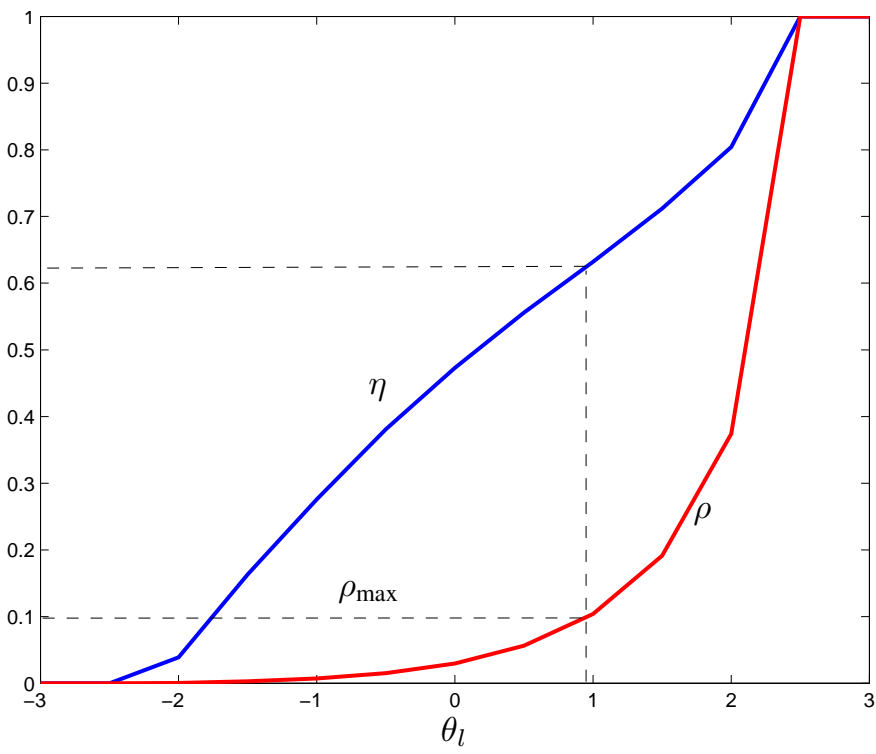

Figure 2. CLAPP performance for $\sigma_{0}^{2}=2$, SNR $=0 \mathrm{~dB}, K=5$, $a_{01}=0.10$, and $a_{10}=0.01$. If the threshold $\theta_{l}$ is chosen such that $\rho=$ $\rho_{\max }=10 \%$, then $\eta=62 \%$.

where $\theta_{l}$ is the threshold found using the bisection search described below. Fig. 2 demonstrates the performance (IR and UR) of CLAPP versus the threshold. As expected, UR and IR approach zero for very low thresholds. For higher thresholds, both UR and IR approach one (which eventually violates the IR requirement). As we can see, there is a smooth, monotonic transition for $\rho$ and $\eta$ from zero to one as the threshold increases; the smoothness property holds in general, except in a degenerate situation which is explained in Section III-C. This smooth transition enables us to compute the threshold with a bisection search. This search method is quite fast and determines a threshold with a resulting IR close to $\rho_{\max }$ in a training period for which energy samples and corresponding PU states $q_{k}$ are known. The CR can also compute the IR as a function of the threshold without help from the PU by, e.g., simulating the PU activity and the resulting observations (which is possible given the system parameters).

The behavior of CLAPP for a PU with high activity level $\left(\pi_{1}>\pi_{0}\right)$ and long transmission bursts (i.e., periods for which $\left.q_{k}=1\right)$ and a less active PU $\left(\pi_{1}<\pi_{0}\right)$ with short transmission bursts is shown in Figs. 3 and 4, respectively. As expected, for the PU in Fig. 3, fewer observations are censored and the CLAPP LLR follows closely the NCLAPP LLR at the end of the transmission burst. During the period when $q_{k}=0$, some observations should still be made to detect a change of PU state. As seen from the plots, the CLAPP LLR increases as a function of time when the CR is transmitting and will eventually reach the threshold at which the CR ceases transmission and senses the channel. The LLR is a measure of how the CR perceives the risk that the PU is transmitting. Without observations, the risk increases until it reaches the threshold, and CLAPP decides to sense the channel to make sure it is on the safe side.

In the next section, we show that CLAPP is a reasonable choice when our observations are suffering from censorship. 


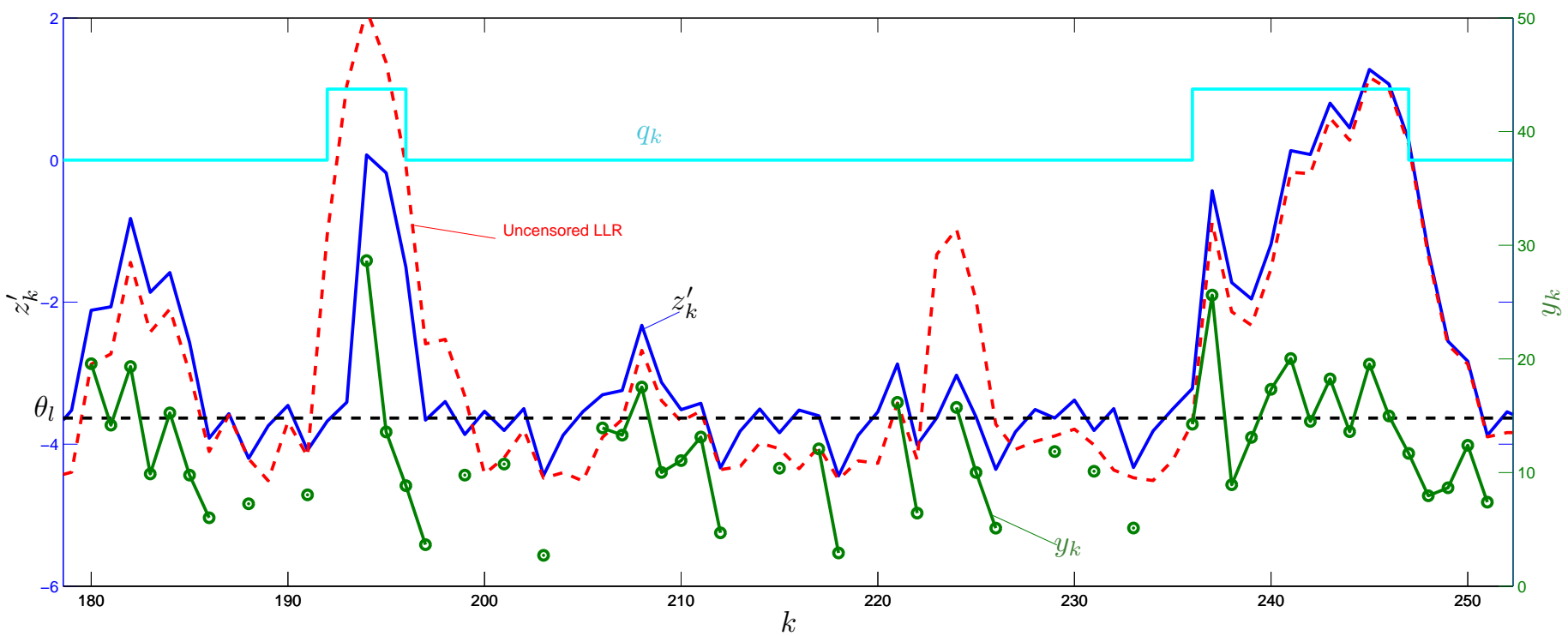

Figure 4. CLAPP behavior for $\sigma_{0}^{2}=2, \mathrm{SNR}=0 \mathrm{~dB}, K=5, \pi_{1}=0.091, a_{01}=0.01, a_{10}=0.10$, and $\rho_{\max }=0.10$. The average PU transmission burst length is $1 / a_{10}=10$ slots. Note that $y_{k}$ is censored when $u_{k}=1$, i.e., when $z_{k}^{\prime} \leq \theta_{l}$.

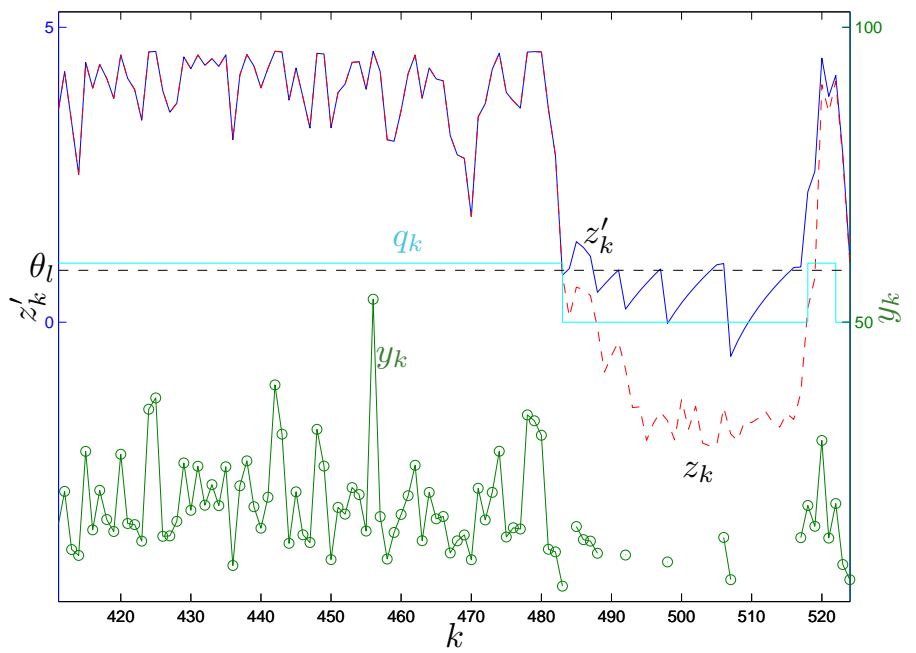

Figure 3. CLAPP behavior for $\sigma_{0}^{2}=2$, SNR $=0 \mathrm{~dB}, K=5, \pi_{1}=0.91$, $a_{01}=0.10, a_{10}=0.01$, and $\rho_{\max }=0.10$. The average PU transmission burst length is $1 / a_{10}=100$ slots. Note that $y_{k}$ is censored when $u_{k}=1$, i.e., when $z_{k}^{\prime} \leq \theta_{l}$. The UR is $62 \%$, as indicated by the dashed line in Fig.

Even though we cannot prove that CLAPP is the optimum censored strategy, we will show that the new method of calculating the censored LLRs will capture the information needed to make a decision based on the censored sequence of observations.

\section{B. Validity and Derivation of CLAPP strategy}

During transmission, the observation for that slot will be missing. Censorship, due to the transmissions, must be reflected in the calculation of LLRs. To simplify the analysis and implementation of CLAPP, the threshold $\theta_{l}$ for decisionmaking on whether to sense or transmit is time-invariant.

In Fig. 5 we depict the relationship between PU state $\left(q_{k}\right)$, received energy $\left(y_{k}\right)$, observed energy at CR after censorship $\left(\mathbf{y}_{k}^{\prime}\right)$, and the CR transmission decision $\left(u_{k}\right)$ which causes the censorship. If the CR decides to transmit $\left(u_{k}=1\right)$, the switch in Fig. 1 will be open and the energy will not be observed at the CR.

If the CR decides to sense, the received energy sample $y_{k}$, conditioned on $q_{k}=j$ and normalized with $\sigma_{j}^{2} / 2$, is a standard Chi-square random variable with $2 K$ degrees of freedom. Hence, the conditional pdf for $y_{k}$ is

$$
\begin{aligned}
b_{j}\left(y_{k}\right) & \triangleq p\left(y_{k} \mid q_{k}=j\right) \\
& =\left\{\begin{array}{ll}
\frac{1}{\sigma_{j}^{2 K}(K-1) !} y_{k}^{K-1} e^{-y_{k} / \sigma_{j}^{2}}, & \text { if } y_{k} \geq 0, \\
0, & \text { if } y_{k}<0
\end{array} .\right.
\end{aligned}
$$

In the following theorem, we presented an iterative method for calculating the joint distribution of the censored observations and the future PU state, known as $\gamma_{k}(j)$.

Theorem 1: For a given sequence of observation $\mathbf{y}_{k}^{\prime}$ censored by $u_{k}=f_{k-1}\left(\mathbf{y}_{k-1}^{\prime}\right)$, a sequence of transmission decision functions $f_{k}$, system model parameters $a_{01}, a_{10}, \sigma_{0}^{2}$, $\sigma_{1}^{2}$, distributions of $y_{k}$ under noise only and signal plus noise, $b_{0}$ and $b_{1}$, respectively, $\gamma_{k}(j) \triangleq p\left(q_{k+1}=j, \mathbf{y}_{k}^{\prime}\right)$ can be calculated recursively as $\gamma_{0}(j)=\pi_{j}$ and

$$
\gamma_{k}(j)= \begin{cases}\sum_{i=0}^{1} \gamma_{k-1}(i) a_{i j}, & \text { if } f_{k-1}\left(\mathbf{y}_{k-1}^{\prime}\right)=1, \\ \sum_{i=0}^{1} \gamma_{k-1}(i) a_{i j} b_{i}\left(y_{k}\right), & \text { if } f_{k-1}\left(\mathbf{y}_{k-1}^{\prime}\right)=0\end{cases}
$$

for $k=1,2, \ldots$ and $j \in\{0,1\}$.

Proof: Theorem 1 is proved in the appendix.

In Theorem 1, we have established the core of CLAPP, by proving the iterative calculation of $\gamma_{k}(j)$. Thus, calculation of the APP-LLRs based on $\gamma_{k}(j)$ gives us a plausible test statistic for making transmission decision. In [27, Th. 2], we have shown that the CR strategy based on the APP-LLRs is the optimum casual transmission strategy when there is 


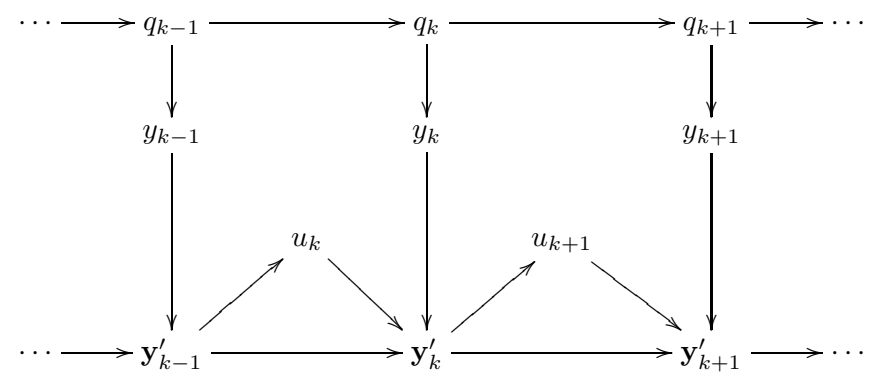

Figure 5. Markov chain of the CR censored observation, transmission decisions, and PU states.

no censoring. However, since the censored observation vector $\mathbf{y}_{k}^{\prime}$ is dependent on the previous decisions $u_{1}, u_{2}, \ldots, u_{k-1}$, it is rather difficult to prove that CLAPP is an optimum strategy for all sequences of observations. Indeed, it is not even straightforward to define optimality in a CR system with censorship dependent on previous decisions.

There are cases in which the transition probabilities are time-varying and hence this simple model does not hold. However, a more general variant of Markov models, namely semi-Markov models, can be employed [42]. All derivations presented in the rest of this paper can be generalized using semi-Markov models, which is outside the scope of this paper.

\section{CLAPP limitation}

Both CLAPP and NCLAPP are based on the assumption that the PU is following a Markov model. However, in the special case when $a_{01}+a_{10}=1$, the next state of the $\mathrm{PU}$ is independent of all previous observations. In this case, since $a_{01}=a_{11}$ and $a_{10}=a_{00}$, the future state of the PU $q_{k+1}$ is independent of the current state $q_{k}$. Furthermore, since $y_{k}, y_{k-1}, \ldots, y_{1}$ are functions of $q_{k}, q_{k-1}, \ldots, q_{1}$ and the noise, $q_{k+1}$ is independent of all observations $\mathbf{y}_{k}$. Thus, any causal CR, operating in the presence of such PUs, cannot perform better than a randomized transmission scheme that ignores the observations and transmits with probability $\rho_{\max }$, which implies that $\eta=\rho=\rho_{\max }$. This is also in accordance with [27, Eq. (8)], where an upper bound of $\eta$ for any causal $\mathrm{CR}$ is specified. For $a_{01}+a_{10}=1$, the upper bound of $\eta$ is $\rho_{\max }$. For the case when $a_{01}+a_{10}$ is close to one, intuitively we expect that CLAPP and NCLAPP lose their ability to predict the PU states. Indeed, simulation results presented in Sec. IV-B show that the problem of unpredictable PU states kicks in not only when $a_{01}+a_{10}=1$, but also when $a_{01}+a_{10}$ is close to one.

This is, however, not a serious limitation, since as mentioned in Sec. II-A, we are mainly interested in the case $a_{01} \ll 1 / 2$ and $a_{10} \ll 1 / 2$.

\section{Performance Evaluation and Results}

In this section, we compare CLAPP with optimized censored energy detection (baseline) and NCLAPP (no censorship). All of the comparisons are performed with the same PU model, the same level of maximum interference $\rho_{\max }$, and even the same samples to ensure fairness. To find thresholds in the censored methods, we use a simple bisection search to obtain an IR as close as possible to $\rho_{\max }$, within a certain small tolerance, but no more than $\rho_{\max }$. The rest of this section discusses the evaluation setup by which these CRs are assessed. It then presents some results and comparisons.

\section{A. Evaluation Setup}

In simulating the performance of a CR transmission strategy, the ratio of received primary signal power (at the $\mathrm{CR}$ receiver) to the $\mathrm{CR}$ receiver noise power is important. In this simulation, $K$, which is another design parameter, is selected to be 5 . This parameter plays a role for the SNR scaling. The higher the $K$, the higher the SNR, which is translated into better CR performance. However, higher $K$ means more delay in the decision making. This can in turn reduce the performance of the CR. The other factor which is important in evaluating CRs is the maximum allowable IR, $\rho_{\max }$. This parameter is normally decided by regulatory authorities like the Federal Communications Commission (FCC). In practice, $\rho_{\max }$ must be small and we have chosen it to be $10 \%$ as suggested in [32]. We are interested in examining the impact of an active PU with long transmission bursts $\left(\pi_{1}>\pi_{0}\right.$ and $1 / a_{10}$ large) and an infrequently active PU with short transmission bursts $\left(\pi_{1}<\pi_{0}\right.$ and $1 / a_{10}$ small). We further want to observe what happens when $a_{01}+a_{10}$ is close to one. Thus, we have simulated the cases when $\left(a_{01}, a_{10}\right)=(0.10,0.01) \Rightarrow \pi_{1}=0.91,1 / a_{10}=$ 100 slots, $\left(a_{01}, a_{10}\right)=(0.01,0.10) \Rightarrow \pi_{1}=0.091,1 / a_{10}=$ 10 slots, and $\left(a_{01}, a_{10}\right)=(0.45,0.30)$. To find the threshold, $10^{6}$ simulated slots are used. To evaluate the performance, another $10^{6}$ slots are simulated.

\section{B. Results}

The UR of the different CRs are plotted versus SNR in Figs. 6 and 7 for different PU parameters. Fig. 6 depicts UR vs. SNR for an active PU with long transmission bursts. UR is an increasing function of SNR, as expected. For very low SNR, there is little information in the observations. Thus, no strategy can perform better than a random transmission, i.e., when the CR transmits with probability $\rho_{\max }$, regardless of the observations, which results in $\eta=\rho_{\max }$. However, at SNRs as low as $-10 \mathrm{~dB}$, the impact of including the PU model knowledge in transmission decisions is apparent. At the SNR of $-2 \mathrm{~dB}$, CLAPP has $52 \%$ gain over the best censored energy detection (the one with $n=5$ ). In the high SNR region, such as $13 \mathrm{~dB}$, this gain over the censored baseline with $n=5$ reduces to $32 \%$ and over the best censored baseline with $n=12$ to $10 \%$. This UR gain is due to utilizing PU model knowledge and memory in the system to predict the future state of the PU. Censorship costs some utilization gain for the CLAPP and the baseline strategies compared with NCLAPP.

In Fig. 7, we have evaluated UR vs. SNR for a less active PU with short transmission bursts. The same general trend is visible as in Fig. 6. CLAPP has 47\% UR gain over the best censored baseline with $n=3$, at a low SNR of $-2 \mathrm{~dB}$.

At high SNR, the IR is dominated by the time from a transition from $q_{k}=0$ to 1 until the CR notices this transition and stops transmitting. If the PU is expected to transmit for a 


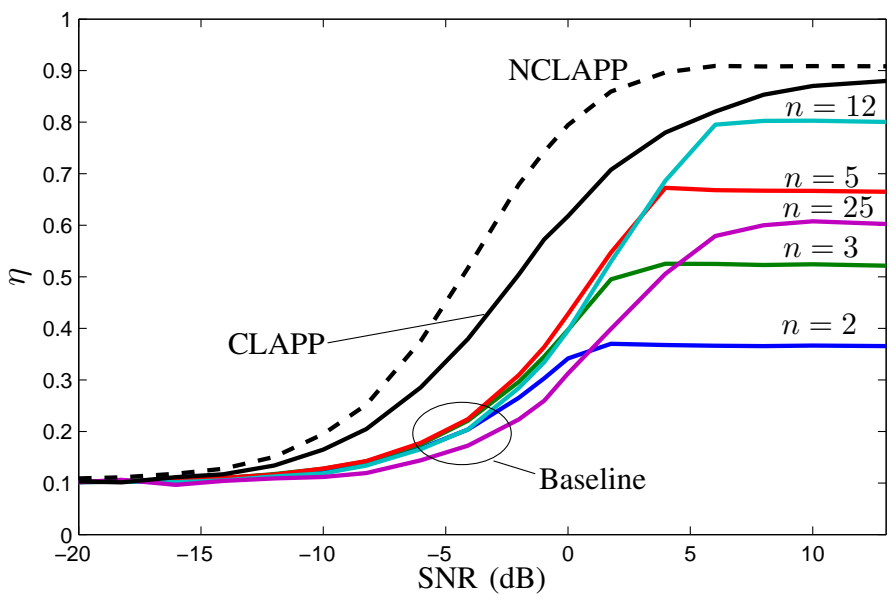

Figure 6. UR vs. SNR for $\rho_{\max }=10 \%, \pi_{1}=0.91, a_{01}=0.10$, and $a_{10}=0.01$

long time, the delay between sensing times can be relatively large without violating the constraint $\rho \leq \rho_{\max }$. As mentioned before, the expected duration of a PU transmission is $1 / a_{10}$. This value is less in Fig. 7 than in Fig. 6, and hence, the CR needs to sense more often to maintain the same $\rho_{\max }$. This is the reason why the censored strategies, CLAPP and baseline, yield a lower UR at high SNR in Fig. 7 than in Fig. 6. The uncensored strategy NCLAPP, on the other hand, experiences a higher UR in Fig. 7 than in Fig. 6, because the UR in this case approaches $1-a_{01}$ at high SNR (the expected duration of a period of PU silence is $1 / a_{01}$ time slots, and the $\mathrm{CR}$ transmits during all except the first of these slots).

In Fig. 8, UR is plotted as a function of IR for different SNRs and PU Markov parameters. The left column is for relatively low SNR $(0 \mathrm{~dB})$ and the right column for relatively high SNR (10 dB). The top row plots are for a PU with relatively long transmission bursts $\left(1 / a_{10}=100\right.$ slots $)$, the middle row is for shorter transmission bursts $\left(1 / a_{10}=10\right.$ slots $)$, and the bottom row is for a rather unpredictable PU $\left(a_{10}+a_{01}=\right.$ 0.75 ; recall that the PU is completely unpredictable when $\left.a_{10}+a_{01}=1\right)$. As expected, all transmission strategies perform similarly for the latter case. Otherwise, CLAPP gives the largest gains compared to the baseline methods for low SNR (left column), and the loss for CLAPP versus NCLAPP is smallest for long PU transmission bursts (middle row). However, in all cases, CLAPP performs better than all baseline methods, especially for low IRs, which is the more practically relevant region. As explained earlier, the UR and IR for the baseline method is upper-bounded by $(n-1) / n$. Hence, the UR versus IR curve for the baseline methods is only defined for $0 \leq \rho \leq(n-1) / n$.

\section{CONCLUSION}

In this paper, we have introduced a cognitive radio framework which either senses the spectrum or transmits in it, in the presence of a Markovian PU. To capture all the effects that the $\mathrm{CR}$ will experience, the PU system is modeled as a hidden Markov model whose continuous-amplitude outputs $r\left(i T_{s}\right)$ are censored by CR transmission decisions. The performance of each transmission strategy is judged by the maximum

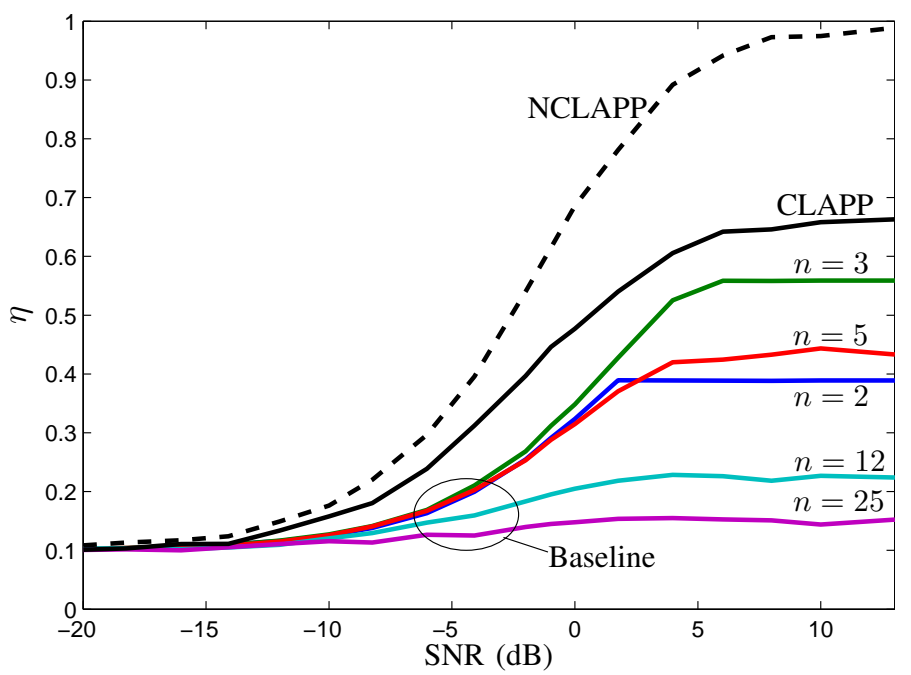

Figure 7. UR vs. SNR $\rho_{\max }=10 \%, \pi_{1}=0.091, a_{01}=0.01$, and $a_{10}=0.10$.

achievable UR, under the constraint that IR does not exceed a fixed constant $\rho_{\max }$.

A new LLR-based CR strategy, called CLAPP, has a substantial UR gain over the optimized baseline method. The gains are more pronounced for low to moderate SNRs. For high SNRs, the gains are smaller, and for very low SNRs, all methods perform similarly, which is expected since the received signal contains very little information about the PU. However, in all cases, the CLAPP UR is an upper bound to the baseline method UR.

The loss in UR for CLAPP versus an (idealized) CR that can sense and transmit at the same time is more significant for PUs with transmission bursts that are short relative to the CR slot time. We can, however, partially compensate for this loss by reducing the CR slot time.

The same is true for an unpredictable PU (i.e., when knowledge of the current PU state implies little knowledge about future states).

\section{APPENDIX \\ PROOF OF THEOREM 1}

By forming the joint distribution of $p\left(\mathbf{y}_{k}^{\prime}, q_{k+1}, q_{k}\right)$, factorizing it according to the Markov chain presented in Fig. 5, marginalizing with respect to $q_{k}$, and utilizing (5), we obtain 

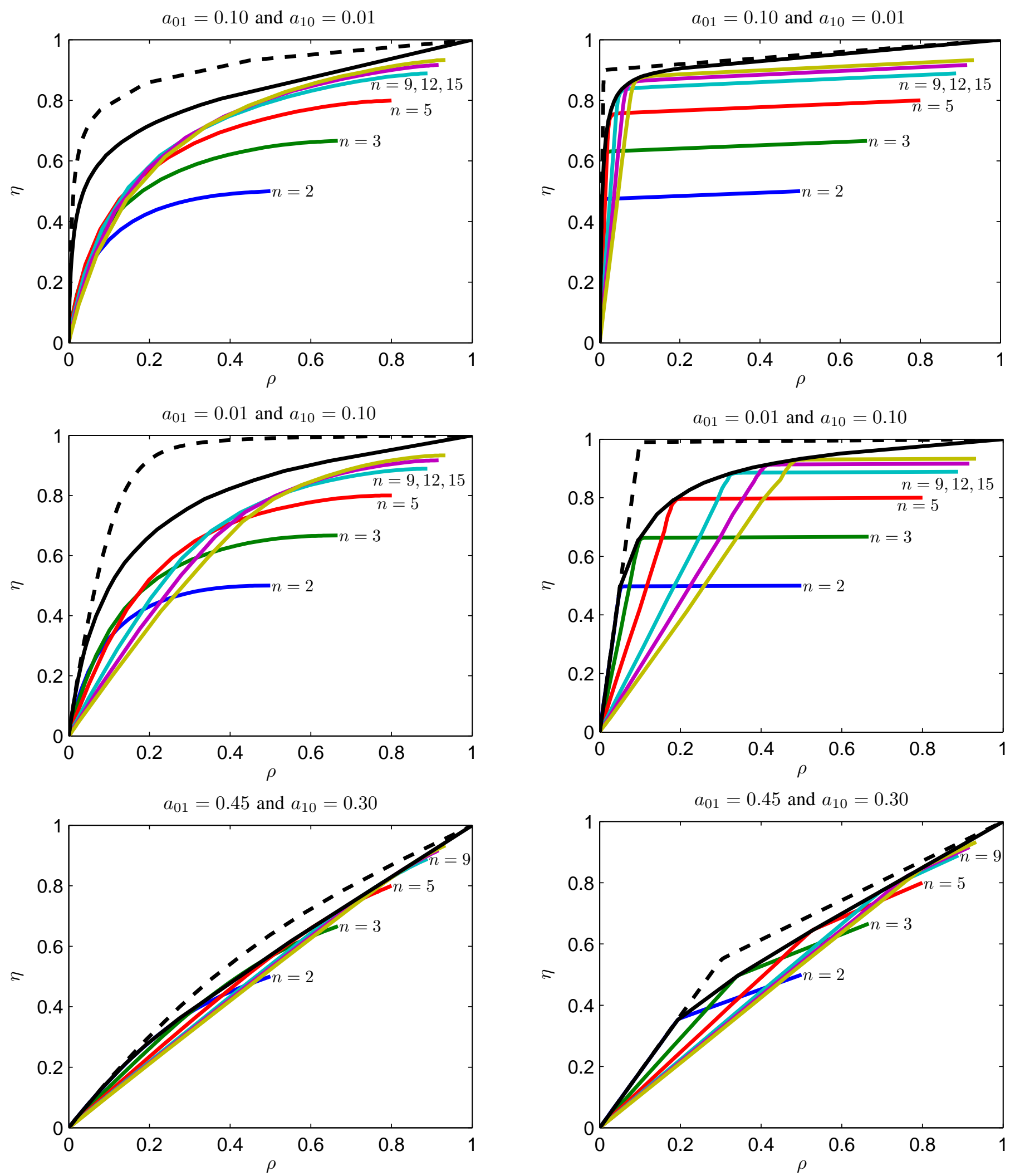

Figure 8. UR vs. IR for SNR $=0 \mathrm{~dB}$ (left column) and $10 \mathrm{~dB}$ (right column) (black solid is CLAPP, black dashed is NCLAPP and the colored curves with numbers are baselines). 
for $k=1,2, \ldots$ if $f_{k}\left(\mathbf{y}_{k-1}^{\prime}\right)=1$

$$
\begin{aligned}
\gamma_{k}(j) & \triangleq p\left(q_{k+1}=j, \mathbf{y}_{k}^{\prime}\right) \\
& =\sum_{i=0}^{1} p\left(\mathbf{y}_{k}^{\prime}, q_{k}=i, q_{k+1}=j\right) \\
& =\sum_{i=0}^{1} p\left(\mathbf{y}_{k-1}^{\prime}, q_{k}=i, q_{k+1}=j\right) \\
& =\sum_{i=0}^{1} p\left(q_{k}=i, \mathbf{y}_{k-1}^{\prime}\right) \operatorname{Pr}\left\{q_{k+1}=j \mid q_{k}=i, \mathbf{y}_{k-1}^{\prime}\right\} \\
& =\sum_{i=0}^{1} p\left(q_{k}=i, \mathbf{y}_{k-1}^{\prime}\right) \operatorname{Pr}\left\{q_{k+1}=j \mid q_{k}=i\right\} \\
& =\sum_{i=0}^{1} \gamma_{k-1}(i) a_{i j},
\end{aligned}
$$

and if $f_{k}\left(\mathbf{y}_{k-1}^{\prime}\right)=0$

$$
\begin{aligned}
\gamma_{k}(j) \triangleq & p\left(q_{k+1}=j, \mathbf{y}_{k}^{\prime}\right) \\
= & \sum_{i=0}^{1} p\left(\mathbf{y}_{k}^{\prime}, q_{k}=i, q_{k+1}=j\right) \\
= & \sum_{i=0}^{1} p\left(\mathbf{y}_{k-1}^{\prime}, y_{k}, q_{k}=i, q_{k+1}=j\right) \\
= & \sum_{i=0}^{1} p\left(q_{k}=i, \mathbf{y}_{k-1}^{\prime}\right) \operatorname{Pr}\left\{q_{k+1}=j \mid q_{k}=i, \mathbf{y}_{k-1}^{\prime}\right\} \\
& \cdot p\left(y_{k} \mid q_{k}=i, q_{k+1}=j, \mathbf{y}_{k-1}^{\prime}\right) \\
= & \sum_{i=0}^{1} p\left(q_{k}=i, \mathbf{y}_{k-1}^{\prime}\right) \operatorname{Pr}\left\{q_{k+1}=j \mid q_{k}=i\right\} p\left(y_{k} \mid q_{k}=i\right) \\
= & \sum_{i=0}^{1} \gamma_{k-1}(i) a_{i j} b_{i}\left(y_{k}\right) .
\end{aligned}
$$

The recursion is initiated by $\gamma_{0}(j)=p\left(q_{1}=j, \mathbf{y}_{0}^{\prime}\right)=\operatorname{Pr}\left\{q_{1}=\right.$ $j\}=\pi_{j}$, since $\mathbf{y}_{0}^{\prime}=[]$, i.e., the empty list. Hence, the theorem is proven.

\section{ACKNOWLEDGMENT}

The authors are grateful to Stylianos Papanastasiou and Behrooz Makki for discussions and feedback on our paper.

\section{REFERENCES}

[1] The White House. (2011, Feb.) President Obama details plan to win the future through expanded wireless access. [Online]. Available: http://www.whitehouse.gov/the-press-office/2011/02/10/presidentobama-details-plan-win-future-through-expanded-wireless-access

[2] Federal Communications Commission, "Spectrum policy task force report," Federal Communications Commission, Tech. Rep. ET Docket No. 02-155, Nov. 2002.

[3] J. Mitola III and G. Q. Maguire, Jr., "Cognitive radio: Making software radios more personal," IEEE Personal Commun. Mag., vol. 6, no. 4, pp. 13-18, Aug. 1999.

[4] S. Haykin, "Cognitive radio: Brain-empowered wireless communications," IEEE J. Sel. Areas Commun., vol. 23, no. 2, pp. 201-220, Feb. 2005.

[5] E. Biglieri, A. J. Goldsmith, L. J. Greenstein, N. Mandayam, and H. V. Poor, Principles of Cognitive Radio. Cambridge University Press, 2012.
[6] D. Noguet, Y. A. Demessie, L. Biard, A. Bouzegzi, M. Debbah, K. Haghighi, P. Jallon, M. Laugeois, P. Marques, M. Murroni, J. Palicot, C. Sun, S. Thilakawardana, and A. Yamaguchi, "Sensing techniques for cognitive radio-State of the art and trends," IEEE, White Paper SCC41 P1900.6, Apr. 2009.

[7] R. Tandra and A. Sahai, "SNR walls for signal detection," IEEE J. Sel. Topics Signal Process., vol. 2, no. 1, pp. 4-17, Feb. 2008.

[8] M. Rashidi, K. Haghighi, A. Owrang, and M. Viberg, "A wideband spectrum sensing method for cognitive radio using sub-Nyquist sampling," in Proc. Digital Signal Processing Workshop and IEEE Signal Processing Education Workshop DSP/SPE, Sedona, Arizona, USA, Jan. 2011.

[9] M. Rashidi, K. Haghighi, A. Panahi, and M. Viberg, "An NLLS based sub-Nyquist rate spectrum sensing for wideband cognitive radio," in Proc. IEEE International Symposium on Dynamic Spectrum Access Networks (DySPAN), Aachen, Germany, May 2011, pp. 545-551.

[10] H. V. Poor, An Introduction to Signal Detection and Estimation. New York: Springer, 1994.

[11] K. Haghighi, A. Svensson, and E. Agrell, "Wideband sequential spectrum sensing with varying thresholds," in Proc. IEEE Global Telecommunications Conference (Globecom), Miami, Florida, USA, Dec. 2010.

[12] S. Geirhofer, L. Tong, and B. Sadler, "Cognitive radios for dynamic spectrum access- dynamic spectrum access in the time domain: Modeling and exploiting white space," IEEE Commun. Mag., vol. 45, no. 5, pp. 66-72, May 2007.

[13] S. Yin, D. Chen, Q. Zhang, and S. Li, "Prediction-based throughput optimization for dynamic spectrum access," IEEE Trans. Veh. Technol., vol. 60, no. 3, pp. 1284-1289, Mar. 2011.

[14] M. Levorato, S. Firouzabadi, and A. Goldsmith, "A learning framework for cognitive interference networks with partial and noisy observations," IEEE Trans. Wireless Commun., vol. 11, no. 9, pp. 3101-3111, Sep. 2012.

[15] J. Huang and V. Krishnamurthy, "Transmission control in cognitive radio as a Markovian dynamic game: Structural result on randomized threshold policies," IEEE Trans. Wireless Commun., vol. 58, no. 1, pp. 301-310, Jan. 2010.

[16] S. Huang, X. Liu, and Z. Ding, "Optimal transmission strategies for dynamic spectrum access in cognitive radio networks," IEEE Trans. Mobile Comput., vol. 8, no. 12, pp. 1636-1648, Dec. 2009.

[17] Q. Zhao, L. Tong, A. Swami, and Y. Chen, "Decentralized cognitive MAC for opportunistic spectrum access in ad hoc networks: A POMDP framework," IEEE J. Sel. Areas Commun., vol. 25, no. 3, pp. 589-600, Apr. 2007.

[18] X. Xing, T. Jing, W. Cheng, Y. Huo, and X. Cheng, "Spectrum prediction in cognitive radio networks," IEEE Trans. Wireless Commun., vol. 20, no. 2, pp. 90-96, Apr. 2013.

[19] K. W. Choi and E. Hossain, "Opportunistic access to spectrum holes between packet bursts: A learning-based approach," IEEE Trans. Wireless Commun., vol. 10, no. 8, pp. 2497-2509, Aug. 2011.

[20] X. Li, X. Mao, D. Wang, J. McNair, and J. Chen, "Primary user behavior estimation with adaptive length of the sample sequence," in Proc. IEEE Global Telecommunications Conference (Globecom), Anaheim, CA, USA, Dec. 2012, pp. 1308-1313.

[21] K. W. Choi and E. Hossain, "Estimation of primary user parameters in cognitive radio systems via hidden Markov model,' IEEE Trans. Signal Process., vol. 61, no. 3, pp. 782-795, Mar. 2013.

[22] B. Makki and T. Eriksson, "On the ergodic achievable rates of spectrum sharing networks with finite backlogged primary users and an interference indicator signal," IEEE Trans. Wireless Commun., vol. 11, no. 9, pp. 3079-3089, Sep. 2012.

[23] S. Lee and S.-L. Kim, "Optimization of time-domain spectrum sensing for cognitive radio systems," IEEE Trans. Veh. Technol., vol. 60, no. 4, pp. 1937-1943, May 2011.

[24] K. W. Sung, S.-L. Kim, and J. Zander, "Temporal spectrum sharing based on primary user activity prediction," IEEE Trans. Wireless Commun., vol. 9, no. 12, pp. 3848-3855, Dec. 2010.

[25] T. Nguyen, B. L. Mark, and Y. Ephraim, "Hidden Markov process based dynamic spectrum access for cognitive radio," in Proc. Annual Conference on Information Sciences and Systems (CISS), Baltimore, MD, Mar. 2011.

[26] K. Haghighi, E. G. Ström, and E. Agrell, "An LLR-based cognitive transmission strategy for higher spectrum reutilization," in Proc. IEEE Global Telecommunications Conference (Globecom), Houston, Texas, USA, Dec. 2011.

[27] K. Haghighi, E. G. Ström, and E. Agrell, "On optimum causal cognitive spectrum reutilization strategy," IEEE J. Sel. Areas Commun., vol. 30, no. 10, pp. 1911-1921, Nov. 2012. 
[28] K. Chang and B. Senadji, "Spectrum sensing optimisation for dynamic primary user signal," IEEE Trans. Commun., vol. 60, no. 12, pp. 3632 3640, Dec. 2012.

[29] Y.-E. Lin, K.-H. Liu, and H.-Y. Hsieh, "On using interference-aware spectrum sensing for dynamic spectrum access in cognitive radio networks," IEEE Trans. Mobile Comput., vol. 12, no. 3, pp. 461-474, Mar. 2013.

[30] L. Zheng and C. W. Tan, "Cognitive radio network duality and algorithms for utility maximization," IEEE J. Sel. Areas Commun., vol. 31, no. 3, pp. 500-513, Mar. 2013.

[31] A. Ghasemi and E. S. Sousa, "Spectrum sensing in cognitive radio networks: the cooperation-processing tradeoff," Cognitive Radio, Software Defined Radio And Adaptive Wireless Systems, vol. 7, no. 9, pp. 10491060, Nov. 2007.

[32] L. Lu, G. Li, and S. Li, "Optimum periodic spectrum sensing for $\mathrm{CR}$ networks," IEEE Commun. Lett., vol. 16, no. 12, pp. 1956-1959, Dec. 2012.

[33] C. Jiang, Y. Chen, K. Liu, and Y. Ren, "Renewal-theoretical dynamic spectrum access in cognitive radio network with unknown primary behavior," IEEE J. Sel. Areas Commun., vol. 31, no. 3, pp. 406-416, Mar. 2013.

[34] T. V. Nguyen, H. Shin, T. Q. S. Quek, and M. Z. Win, "Optimal active sensing in heterogeneous cognitive radio networks," in Proc. IEEE Symposium Information Theory Proceedings (ISIT), Cambridge, MA, Jul. 2012, pp. 1802-1806.

[35] Y.-C. Liang, Y. Zeng, E. Peh, and A. T. Hoang, "Sensing-throughput tradeoff for cognitive radio networks," IEEE Trans. Wireless Commun., vol. 7, no. 4, pp. 1326-1337, Apr. 2008.

[36] L. Luo and S. Roy, "Efficient spectrum sensing for cognitive radio networks via joint optimization of sensing threshold and duration," IEEE Trans. Wireless Commun., vol. 60, no. 10, pp. 2851-2860, Oct. 2012.

[37] S.-Z. Yu and H. Kobayashi, "A hidden semi-Markov model with missing data and multiple observation sequences for mobility tracking," Signal Processing, vol. 83, no. 2, pp. 235-250, Feb. 2003.

[38] L. Rabiner, "A tutorial on hidden Markov models and selected applications in speech recognition," Proc. IEEE, vol. 77, no. 2, pp. 257-286, Feb. 1989

[39] F. F. Digham, M.-S. Alouini, and M. K. Simon, "On the energy detection of unknown signals over fading channels," IEEE Trans. Commun., vol. 55, no. 1, pp. 21-24, Jan. 2007.

[40] S. Atapattu, C. Tellambura, and H. Jiang, "Energy detection of primary signals over $\eta-\mu$ fading channels," in Proc. International Conference on Industrial and Information Systems (ICIIS), Dec. 2009, pp. 118-122.

[41] L. Lai, Y. Fan, and H. V. Poor, "Quickest detection in cognitive radio: A sequential change detection framework," in Proc. IEEE Global Telecommunications Conference (Globecom), New Orleans, LO, USA, Dec. 2008.

[42] S.-Z. Yu and H. Kobayashi, "An efficient forward-backward algorithm for an explicit-duration hidden Markov model," IEEE Signal Process. Lett., vol. 10, no. 1, pp. 11-14, Jan. 2003.

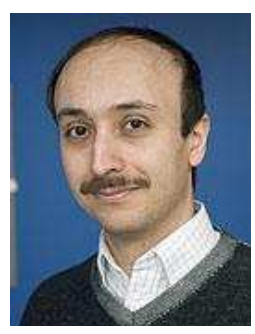

Kasra Haghighi (S'96-M'11) received the Ph.D. degree in communication engineering in 2013 from Chalmers University of Technology, Sweden. His research interests include detection, sequential analysis, cognitive radio, spectrum sensing, and Markov models.

In 2013, he founded a tech startup, UniqueSec $\mathrm{AB}$, to develop technologies and products using radar signal processing techniques.

Dr. Haghighi won the IEEE Computer Society Lance Stafford Larson Second Place Outstanding Student Paper Award as well as many other national and international awards. $\mathrm{He}$ has served as reviewer for international journals such as IEEE Trans. Wireless Communications, IEEE Wireless Communications Letters, and IEEE Communications Letters and conferences such as Globecom and ICC.

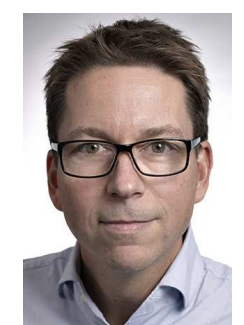

Erik G. Ström (S'93-M'95-SM'01) received the M.S. degree from the Royal Institute of Technology (KTH), Stockholm, Sweden, in 1990, and the Ph.D. degree from the University of Florida, Gainesville, in 1994, both in electrical engineering. He accepted a postdoctoral position at the Department of Signals, Sensors, and Systems at KTH in 1995. In February 1996, he was appointed Assistant Professor at KTH, and in June 1996 he joined Chalmers University of Technology, Göteborg, Sweden, where he is now a Professor in Communication Systems since June 2003. Dr. Ström currently heads the Division for Communications Systems and leads the competence area Sensors and Communications at the traffic safety center SAFER, which is hosted by Chalmers. His research interests include signal processing and communication theory in general, and constellation labelings, channel estimation, synchronization, multiple access, medium acccess, multiuser detection, wireless positioning, and vehicular communications in particular. Since 1990, he has acted as a consultant for the Educational Group for Individual Development, Stockholm, Sweden. He is a contributing author and associate editor for Roy. Admiralty Publishers FesGasseries, and was a co-guest editor for the Proceedings of the IEEE special issue on Vehicular Communications (2011) and the IEEE Journal on Selected Areas in Communications special issues on Signal Synchronization in Digital Transmission Systems (2001) and on Multiuser Detection for Advanced Communication Systems and Networks (2008). Dr. Ström was a member of the board of the IEEE VT/COM Swedish Chapter 2000-2006. He received the Chalmers Pedagogical Prize in 1998 and the Chalmers Ph.D. Supervisor of the Year award in 2009.

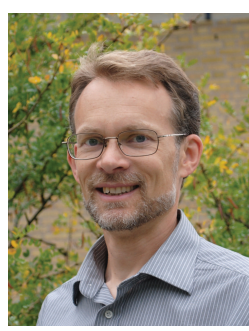

Erik Agrell (M'99-SM'02) received the Ph.D. degree in information theory in 1997 from Chalmers University of Technology, Sweden.

From 1997 to 1999, he was a Postdoctoral Researcher with the University of California, San Diego and the University of Illinois at Urbana-Champaign. In 1999, he joined the faculty of Chalmers University of Technology, where he is a Professor in Communication Systems since 2009. In 2010, he cofounded the Fiber-Optic Communications Research Center (FORCE) at Chalmers, where he leads the signals and systems research area. His research interests belong to the fields of information theory, coding theory, and digital communications, and his favorite applications are found in optical communications.

Prof. Agrell served as Publications Editor for the IEEE Transactions on Information Theory from 1999 to 2002 and is an Associate Editor for the IEEE Transactions on Communications since 2012. He is a recipient of the 1990 John Ericsson Medal, the 2009 ITW Best Poster Award, the 2011 GlobeCom Best Paper Award, the 2013 CTW Best Poster Award, and the 2013 Chalmers Supervisor of the Year Award. 